\title{
A simple power supply and control unit for pulsed operation of a microchannel plate imaging detector
}

\author{
J UPADHYAY, H R BUNDEL, R CHANDRA, J A CHAKERA, C P NAVATHE \\ and P D GUPTA
}

Laser Programme, Centre for Advanced Technology, PO CAT, Indore 452 013, India

e-mail: janky@cat.ernet.in

MS received 2 January 1998; revised 12 October 1998

\begin{abstract}
A simple power supply and control electronic system to operate microchannel plate (MCP) detectors for imaging transient events such as X-ray emission from laser-produced plasmas is described. The power supply consists of two parts, viz. a high voltage pulse generator of $1 \mathrm{kV}$ amplitude with a variable pulse duration of $100 \mu \mathrm{s}$ to $1 \mathrm{~ms}$ for the MCP input, and a regulated $5 \mathrm{kV}$ d.c. for biasing the phosphor screen. The control unit synchronizes the high voltage pulse with the event and operates a safety switch to guard the detector against any accidental rise in the background pressure. A signal-to-noise ratio $\geq 75$ is obtained, limited by the noise contribution of the Charge Coupled Device (CCD)-frame grabber combination attached to the MCP. The system is compact, has provision for both positive and negative pulses, is extendable to higher voltages for operating two-stage $\mathrm{MCP}$, and is immune to electromagnetic interference.
\end{abstract}

Keywords. Microchannel plate; X-ray imaging; pulsed high voltage supply.

\section{Introduction}

Microchannel plates (MCP) are secondary electron multipliers used for detection and imaging of XUV-soft X-ray radiation, electrons and ions (Morgan 1985). Their capability to provide high gain $\left(\geq 10^{4}\right), 2 \mathrm{D}$-imaging with spatial resolution of $20-30 \mu \mathrm{m}$, fast time response, and features like low power consumption, small size, light weight, and immunity to magnetic fields (Hamamatsu Technical Manual 1987) make them very useful in many research and applied areas, such as laser-produced plasmas, magnetically confined plasmas, beam-plasma interaction, mass spectroscopy, optical and X-ray streak cameras, electron microscopy, high energy physics etc. A high voltage of $\sim 1 \mathrm{kV}$, applied across its electrodes, provides gain through secondary electron multiplication. These secondary electrons are accelerated to about $5 \mathrm{kV}$ on to a phosphor screen. The luminous image on the phosphor 
screen is captured by a CCD-frame grabber combination, facilitating on-line analysis of the event under detection.

It is desirable to operate the above detector system with a high signal-to-noise ratio. In detection and imaging of transient events of short durations of a few nanoseconds to microseconds, dark current contribution of the MCP gets integrated over the $20 \mathrm{~ms}$ duration of a single frame of the CCD camera. This deteriorates the signal-to-noise ratio and adversely affects image quality. This problem is circumvented by applying a high voltage gate pulse on the MCP and temporally synchronizing it to include the event under detection. This is also desirable for longer operational life of the MCP. On the other hand, a d.c. voltage can be constantly applied on the phosphor screen. In this note, we present a simple power supply and control electronic system to operate an open-ended MCP detector for imaging of transient events. This includes a regulated power supply for biasing of phosphor screen, a high voltage pulse generator for gating the MCP, and a safety interlock against any accidental increase in the background pressure that may damage the MCP detector. System performance is tested by imaging $\mathrm{X}$-ray emission from plasma produced by a $2 \mathrm{GW}, 28 \mathrm{~ns} \mathrm{Nd}$ : glass laser. The power supply and its control unit are compact, made using indigenously available components, and are immune to electromagnetic interference. These can also be used for pulsed operation of image intensifier tubes. Further, the power supply can be easily modified for negative high voltage pulses and also extended to higher voltages for operating two-stage MCPs.

\section{Description of the system}

\subsection{The system requirement}

A typical experimental setup using MCP for imaging transient events is shown in figure 1 . A high power $\mathrm{Nd}$ : glass laser beam ( $2 \mathrm{GW}, 28 \mathrm{~ns}$ FWHM) is focussed on a planar solid target kept in the centre of a plasma chamber evacuated to $10^{-5}$ torr*. This produces a high temperature plasma of typical dimensions of 100 to $200 \mu \mathrm{m}$ diameter. X-ray emission from this plasma is imaged on the MCP input surface using an X-ray pinhole camera set-up. The MCP in this camera is a single-stage open-ended detector of $25 \mathrm{~mm}$ diameter. It comprises a large number of channels, each of $\sim 20$ micron diameter and $\sim 0.5 \mathrm{~mm}$ length, and provides a gain of $\sim 10^{4}$ at an applied voltage of $1 \mathrm{kV}$ across its two ends. It has a high output impedance of $\sim 1000 \mathrm{M} \Omega$. A visible image is formed on the phosphor screen which is recorded using a CCD camera and a frame-grabber combination. The trigger signal used for firing the laser chain is generated from the laser control unit and is given to the frame-grabber as well as to the MCP power supply.

The MCP requires a high voltage pulse with amplitude adjustable from 200 to $1000 \mathrm{~V}$ and duration varying from 100 to $1000 \mu \mathrm{s}$. This range of pulse duration is governed by the consideration of reducing the dark current contribution of the MCP to the recorded image, as well as for ease of switching circuit design. Next, the polarity of the pulse may be either positive or negative depending on the configuration of the MCP and the application. Quite often, a positive high voltage pulse on the MCP anode is required for Xray detection. However, sometimes a negative high voltage pulse is preferred on the MCP

\footnotetext{
${ }^{*} 1$ torr $=1.01325 \times 10^{5} \mathrm{~Pa}$ at STP
} 


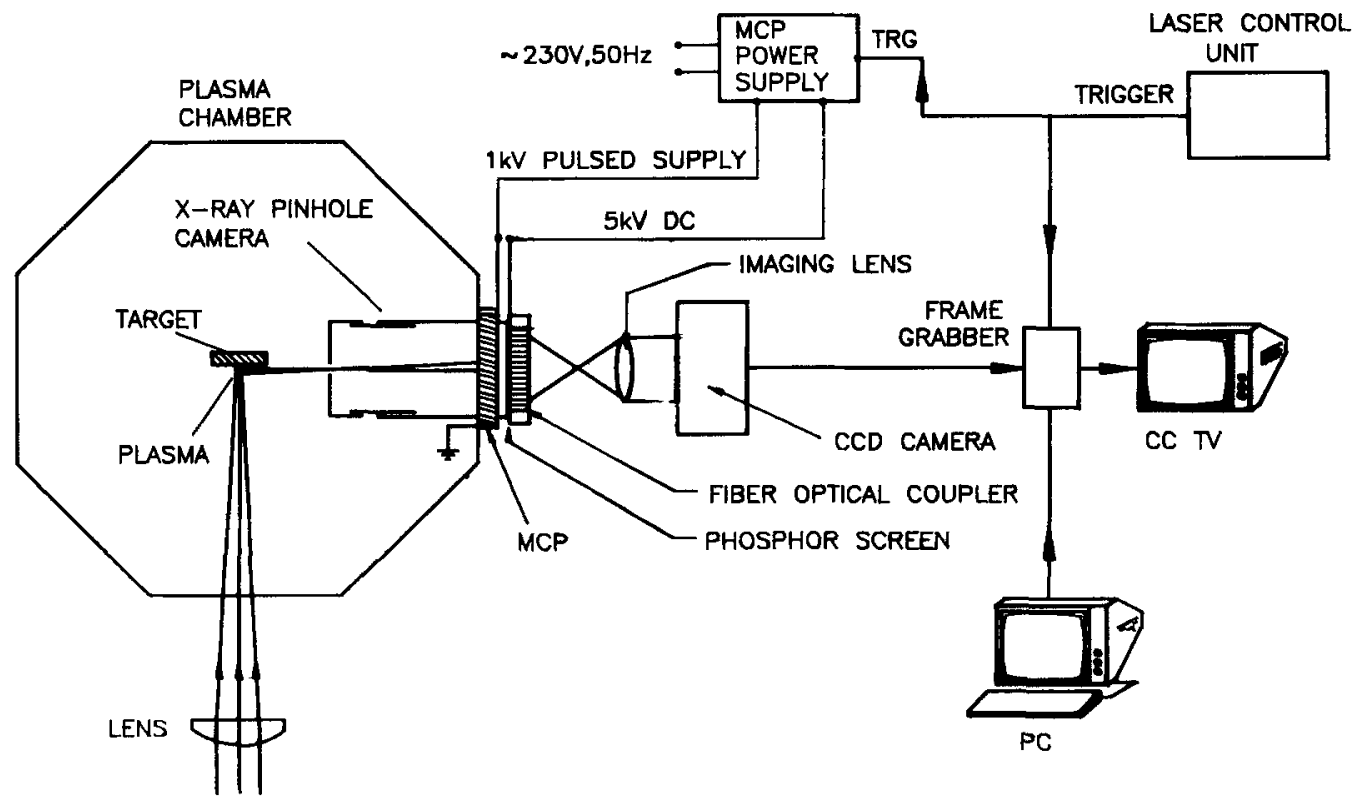

Figure 1. Schematic diagram of the experimental set-up using MCP detector for X-ray imaging of laser-produced plasmas.

cathode for detection of positive ions. Accordingly, the d.c. power supply required for this pulse generator, should have a provision for giving either positive or negative voltage. Further, the high voltage pulse appearing on the MCP should be temporally synchronized to include the event under detection. In addition to the high voltage pulse generator, a $5 \mathrm{kV}$ d.c. is required to accelerate the multiplied electrons from the MCP output onto the phosphor screen to generate a visible image. Finally, from the safety point of view, a safety interlock is required to switch off the power supply automatically if the pressure in the plasma chamber exceeds $10^{-5}$ torr to prevent any damage to the MCP. The various power supplies and control circuits involved are described in the following subsections.

\subsection{Power supply for the phosphor screen}

A schematic diagram of the power supply for the phosphor screen is shown in figure 2 . An astable multivibrator (LM 555) generates a train of pulses at $40 \mathrm{kHz}$. The output of this oscillator is used to generate two complementary pulses. These pulses are applied to two MOSFET switches (IRF 531) viz. $\mathrm{Q}_{1}$ and $\mathrm{Q}_{2}$, connected in a push-pull mode. The specifications for these MOSFETs are $V_{\mathrm{BR}}=60 \mathrm{~V}, I_{\mathrm{D}(\mathrm{ON})}=14 \mathrm{~A}, r_{\mathrm{ds}(\mathrm{on})}=180 \mathrm{~m} \Omega$, $P_{\mathrm{D}(\max )}=75 \mathrm{~W}, V_{\mathrm{gs}(\max )}=4 \mathrm{~V}, \operatorname{Tr}(\max )=75 \mathrm{~ns}, \operatorname{Tf}(\max )=45 \mathrm{~ns}$. The two MOSFETs are alternately switched on and off and switch the current through the primary of a pulse transformer with a ferrite core. This voltage appearing at the secondary is then rectified and filtered to generate $5 \mathrm{kV}$ d.c. voltage. As such, the current requirement for this supply is very small due to a very large impedance of $\mathrm{MCP}(\sim 1000 \mathrm{M} \Omega$ ). However, a load of $\sim 50 \mu \mathrm{A}$ arises predominantly due to the metering requirement. For load regulation, a voltage regulator (LM 723) is used to which an output voltage sensed by a 


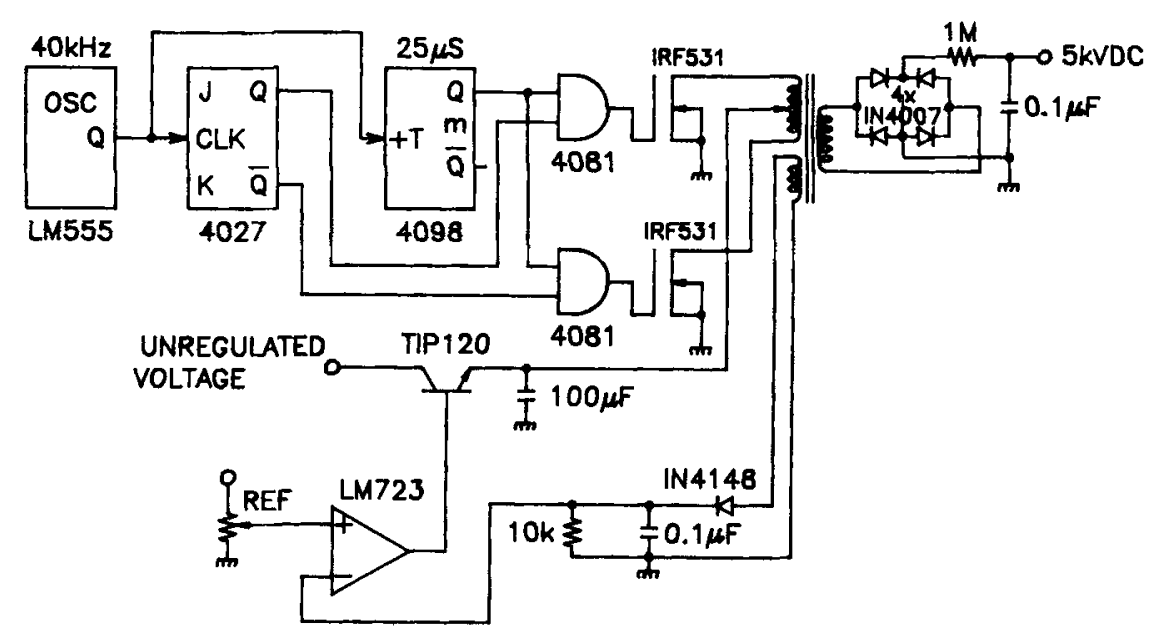

Figure 2. Circuit diagram of $5 \mathrm{kV}$ d.c. power supply for the phosphor screen.

feedback winding is supplied. Feedback signal from a separate winding is preferred over that from high voltage resistors as the latter are quite expensive and difficult to procure. Moreover, the use of feedback winding is convenient for getting either a positive or negative d.c. voltage(required for high voltage pulse generation described in the next section). Design calculations for the feedback voltage and the output voltage at the secondary of the transformer are as follows.

If the period of oscillation at the gate of MOSFETs is $T$, then for $0<t<T / 2, \mathrm{Q}_{1}$ is on and $\mathrm{Q}_{2}$ is off. The regulated voltage $V_{r}$ applied at the centre tap of the primary of the transformer is switched through $\mathrm{Q}_{1}$ resulting in a pulse of peak amplitude $V_{r}$ at its upper half winding. Further, because of the transformer action, the same voltage appears in the other half of the winding. Thus,

$$
V_{1}=V_{2}=+V_{r},
$$

and

$$
V_{0}=\left(+n_{2} / n_{1}\right) V_{1}=\left(+n_{2} / n_{1}\right) V_{r} .
$$

In the second half of the oscillation i.e. $T / 2<t<T, \mathrm{Q}_{1}$ gets off and $\mathrm{Q}_{2}$ gets on. This will switch the current through MOSFET $\mathrm{Q}_{2}$ and will produce a voltage of $\left(V_{2}=-V_{r}\right)$ at the lower half of the primary winding. Similar to the first half cycle,

$$
V_{1}=V_{2}=-V_{r},
$$

and

$$
V_{0}=\left(n_{2} / n_{1}\right) V_{1}=\left(-n_{2} / n_{1}\right) V_{r}
$$

The peak output voltage is then given to a number of multiplier sections to generate the desired d.c. voltage,

$$
V_{\mathrm{dc}}=N \cdot\left(n_{2} / n_{1}\right) V_{r},
$$

where $N$ is the number of multiplier sections. 
In the present case, the reference voltage $V_{r}$ can be varied from 5 to $30 \mathrm{~V}$. The transformer is wound on ferrite $E$-I core with $n_{1}=25, n_{2}=800$ and $n_{3}$ (feed back winding) $=7$. There are 6 multiplier sections. Thus for a d.c. supply voltage of $\sim 26 \mathrm{~V}$ used in this circuit, the output d.c. voltage would be $5 \mathrm{kV}$. Further, by varying the reference voltage, the output d.c. voltage can be varied from $1 \mathrm{kV}$ to $6 \mathrm{kV}$. Next, the voltage developed in the feedback winding is given as

$$
V_{f}=\left(n_{3} / n_{1}\right) V_{r}-V_{d}=(7 / 25) \times 25-0.7=6.3 \mathrm{~V},
$$

where $V_{d}$ is the forward voltage drop across the diode in the feedback path.

The reference voltage is derived from internal reference of LM723, and fed to the noninverting input of the opamp (operational amplifier). The feedback is fed to the inverting input. Thus, for a given setting of the reference voltage, the opamp in LM723 adjusts the value of $V_{r}$ such that $V_{f}$ equals the reference voltage. The effect of diode voltage drop is nullified due to the high gain of the opamp.

To avoid any short circuiting of the supply voltage, a delay (i.e. dead time) of $25 \mu \mathrm{s}$ is introduced between the two gate pulses by using a monoshot (M). Moreover, a separate oscillator (LM 555) has been used to have a better control over driving pulses by varying the frequency and the pulse duration. Load regulation and the ripple voltage are measured to be $0.8 \%$ and $20 \mathrm{~V}$ peak-to-peak respectively in this power supply.

\subsection{High voltage pulser circuit for $M C P$}

This circuit is used to generate the high-voltage pulse required for gating of MCP. For this purpose, we start with a power supply of $1 \mathrm{kV}$, which is essentially similar to the one described above, except for a smaller number of multiplier sections. A schematic of the pulser for positive pulse generation is shown in figure 3 .

In order to generate a pulse of required specifications, the d.c. voltage is applied to a pair of two silicon controlled rectifiers (SCRs) (T30 NU1200DU), one connected in series and the other connected in parallel to the load. These SCRs have a voltage $\left(V_{\mathrm{DRM}}\right)$ and current $\left(I_{\mathrm{AV}}\right)$ of $1200 \mathrm{~V}$ and $30 \mathrm{~A}$ respectively. Their $\mathrm{d} v / \mathrm{d} t$ rating, holding current and latching

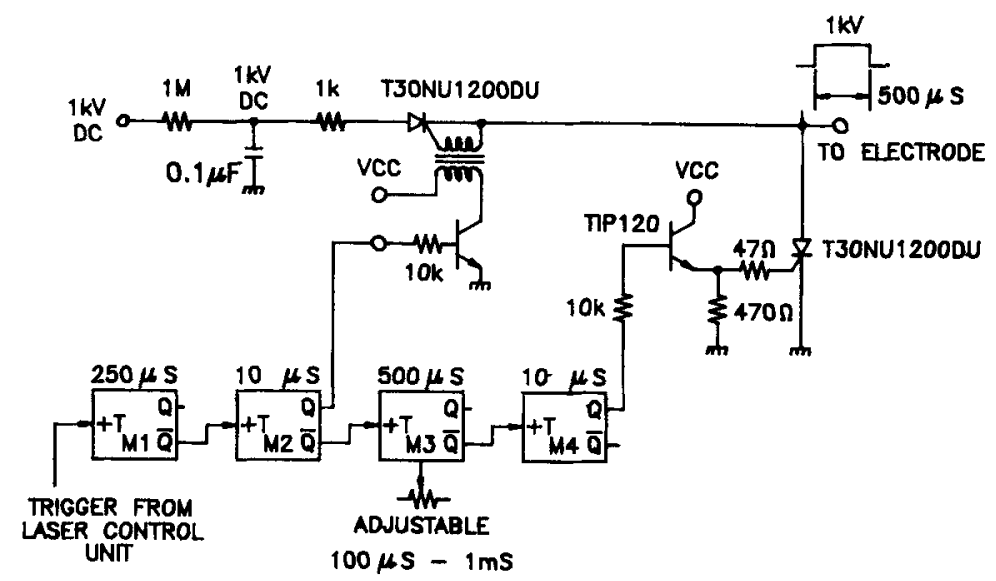

Figure 3. Circuit diagram of high voltage $(+1 \mathrm{kV})$ pulse generator. 


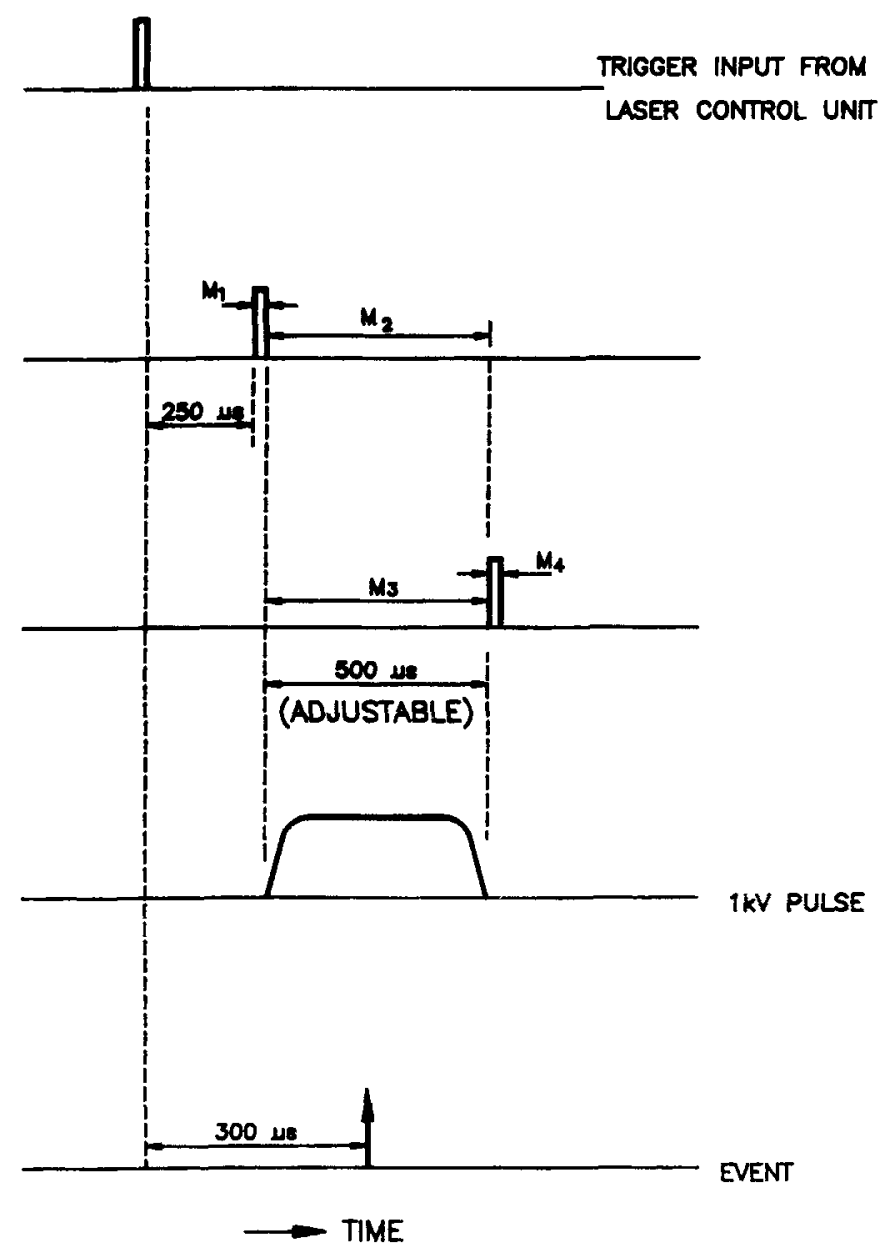

Figure 4. Time sequence of switching monoshots to generate high voltage pulses.

current are $200 \mathrm{~V} / \mu \mathrm{s}, 200 \mathrm{~mA}$ and $400 \mathrm{~mA}$ respectively. The trigger from the laser control unit triggers the monoshot $M 1$ (CD 4098) which generates a delay of $250 \mu \mathrm{s}$ and triggers the monoshot $M 2$, used to fire the series SCR. After this, monoshot $M 3$ is triggered which in turn generates a pulse whose delay is adjustable with the external potentiometer. This delayed pulse at its end triggers the monoshot $M 4$ and the shunt SCR. Thus, a pulse of amplitude equal to the d.c. voltage and duration determined by the monoshot $M 3$ appears at the output. A time sequence of the above switching events is schematically shown in figure 4. The pulse was measured using a Tektronix probe P6015 \& L\&T Gould oscilloscope model 4074 and a typical pulse output as shown in figure 5 .

As per the specifications of the monoshots $\mathrm{CD} 4098$, the variation in pulse duration is limited to a maximum of $2.5 \%$ over the temperature range of $-55^{\circ} \mathrm{C}$ to $+125^{\circ} \mathrm{C}$, and $0.5 \%$ for a power supply variation of $5 \%$. Although device-to-device variation may exist, a given device does not exhibit shot-to-shot variation of more than $1 \%$. In any case, it is possible to adjust the pulse width with the help of an oscilloscope once and for all. It may 


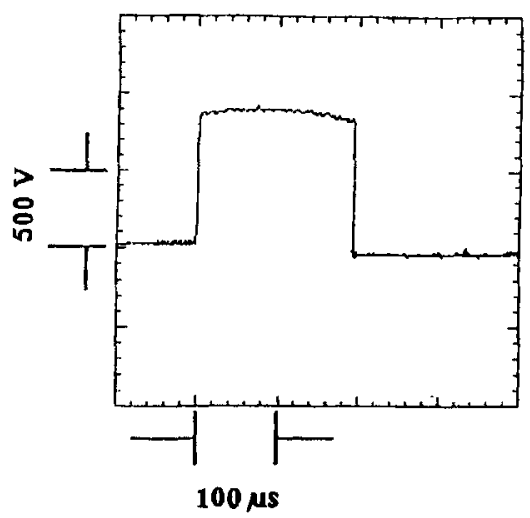

Figure 5. A typical high voltage gating pulse for MCP.

also be noted from figure 3 that for the monoshots $M 2$ and $M 4$, pulse width is not critical. Typically, the maximum duration of the laser plasma events is a few tens of nanoseconds. Hence sufficiently large tolerance levels are available for the settings.

The above scheme for the generation of high voltage positive pulse can also be used to generate high voltage negative pulse by making some simple modifications. The circuit diagram for negative pulse generation is shown in figure 6 . The negative $1 \mathrm{kV}$ d.c. required for this purpose is obtained by reversing the diode polarity in the rectifier section. Further, the SCR polarity is also reversed in the switching section. Moreover, as both the gates are now isolated from the ground, the two SCRs require trigger transformers for triggering. This is in contrast to the positive pulse generator where only one trigger transformer was required for triggering the series SCR.

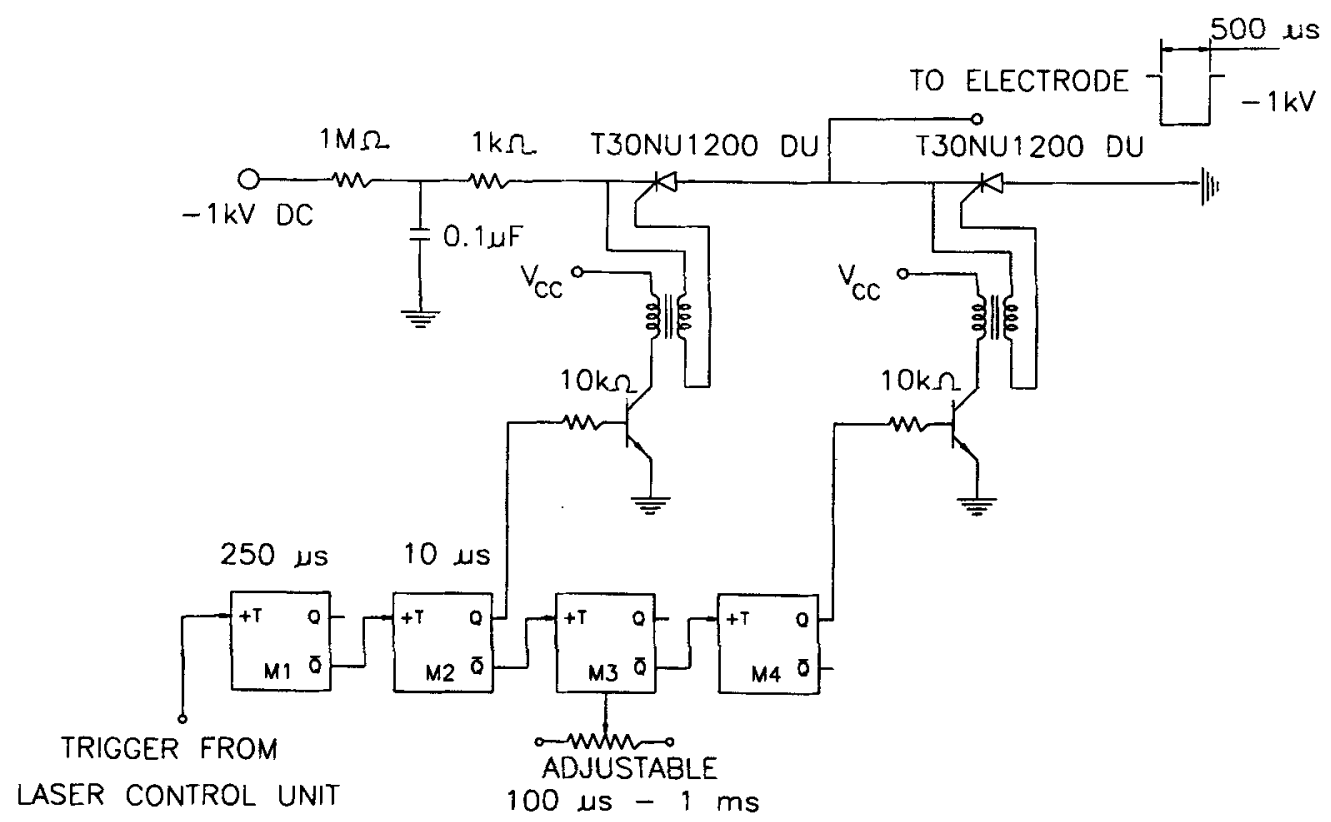

Figure 6. Circuit diagram of high voltage $(-1 \mathrm{kV})$ pulse generator. 


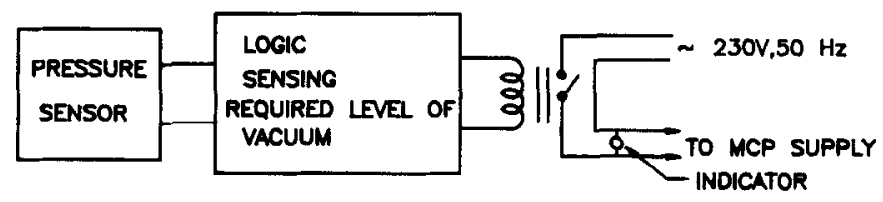

Figure 7. Block diagram of the safety interlock against increase of the background pressure.

In the above scheme, both the pulse width and the amplitude of the output voltage are controllable. By varying the time constant of the monoshot $M 3$, pulse width can be changed from 100 to $1000 \mu \mathrm{s}$ whereas by varying the reference input of the regulator (LM 723) in the d.c. power supply, amplitude of the pulse can be varied from 200 to $1000 \mathrm{~V}$. Further, fast rise and fall times are achieved because of the fast-switching SCRs. The rise and fall times of the output pulse are observed to be $\sim 5 \mu \mathrm{s}$ each . Further, the complete circuit is designed around CMOS logic. This is done to prevent any electromagnetic interference which is common in high power laser laboratories.

\subsection{Safety interlock}

MCP detectors are recommended for use in systems evacuated to pressures $<10^{-5}$ Torr to avoid damage/degradation of the MCP channels. In view of this, a safety interlock has been incorporated to switch off the power supplies in case the chamber pressure exceeds this value. A block diagram of the safety interlock is shown in figure 7. Output of this logic unit operates a relay to switch on output of $230 \mathrm{~V}, 50 \mathrm{~Hz}$ only if the pressure is below a preset level, which in turn is used to power the MCP power supply.

\subsection{Operation and performance}

Performance of the system has been tested by recording X-ray images of laser-produced copper plasma. Figure 8 shows $X$-ray emission region of the plasma produced at a laser intensity of $\sim 2 \times 10^{12} \mathrm{~W} / \mathrm{cm}^{2}$. Intensity versus position scan of this image in a direction parallel to the target plane is shown in figure 9 . A background of 1-2 counts is observed, which is essentially the same as that of a blank frame of the CCD camera without any

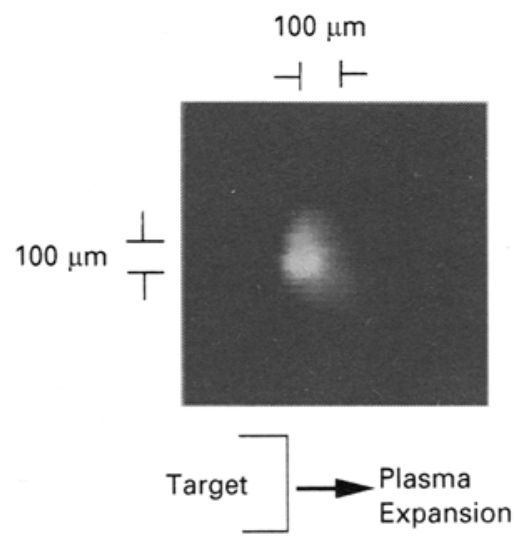

Figure 8. X-ray image of a laser produced copper plasma. 


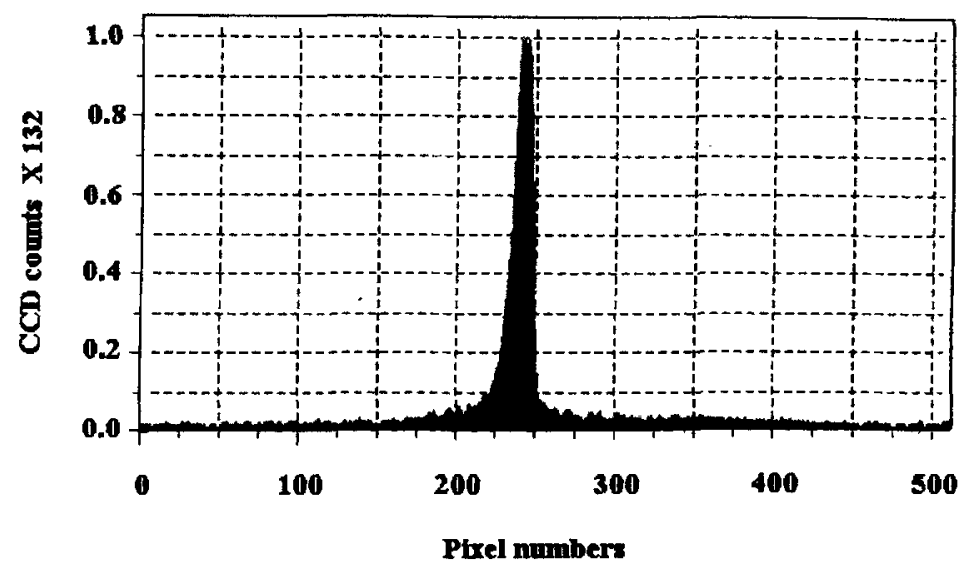

Figure 9. Intensity versus position scan of the X-ray image.

activated MCP. For the CCD camera used in this set-up, the signal is observed to be linear up to about 150 counts. Thus a maximum value of signal to noise ratio $\geq 75$ is obtained which is governed by the CCD camera noise. The pulsed operation of MCP helps in reducing its dark current contribution to the overall noise so that the observed signal-tonoise is essentially limited by that of the CCD camera frame-grabber recording system. The contrast ratio of $\geq 75$ in the present case is quite adequate for obtaining information on spatial profiles of $X$-ray emission relevant to laser-plasma interaction experiments. The power supply and control units are observed to function smoothly without any effect of electromagnetic interference arising due to high power laser operation.

\section{Conclusions}

In conclusion, a simple power supply and control system to operate microchannel plate detectors for imaging transient events is described. The overall electronics is compact and operates smoothly without any electromagnetic interference. This can also be used for pulsed operation of image intensifier tubes. Further, the pulsed MCP supply can be upgraded to higher voltages for operating two-stage MCP detectors simply by increasing the multiplier sections.

\section{References}

Hamamatsu Technical Manual 1987 Characteristics and application of microchannel plates. RES0795 \& T-114

Morgan B L (ed.) 1985 Photoelectronic images devices (London: Academic Press) 